\title{
The Depressed Brain: An Evolutionary Systems Theory
}

Paul B. Badcock ${ }^{1,2,3}$

E-mail: pbadcock@unimelb.edu.au; Ph: +61450211976

Christopher G. Davey ${ }^{1,3}$

E-mail: c.davey@unimelb.edu.au; Ph: +61 403058343

Sarah Whittle 2,4

E-mail: swhittle@unimelb.edu.au; Ph: +61 402597590

Nicholas B. Allen ${ }^{5}$

E-mail: nallen3@uoregon.edu; Ph: +1 5413464075

Karl J. Friston ${ }^{6}$ (senior author)

E-mail: k.friston@ucl.ac.uk; Ph: +44 2034567890

${ }^{1}$ Centre for Youth Mental Health, The University of Melbourne, Melbourne,

Australia, 3052

${ }^{2}$ Melbourne School of Psychological Sciences, The University of Melbourne,

Melbourne, Australia, 3010

${ }^{3}$ Orygen, The National Centre of Excellence in Youth Mental Health, Melbourne, Australia, 3052

${ }^{4}$ Melbourne Neuropsychiatry Centre, Department of Psychiatry, The University of Melbourne and Melbourne Health, Melbourne, Australia, 3053

${ }^{5}$ Department of Psychology, University of Oregon, Eugene, Oregon, USA, 97403

${ }^{6}$ Wellcome Trust Centre for Neuroimaging, University College London, London, UK, WC1N3BG

*Correspondence: pbadcock@unimelb.edu.au (P.B. Badcock) 
ABSTRACT

2 Major depressive disorder is a debilitating condition characterised by diverse

3 neurocognitive and behavioural deficits. Nevertheless, our species-typical capacity for

4 depressed mood implies that it serves an adaptive function. Here, we apply an

5 interdisciplinary theory of brain function to explain depressed mood and its clinical

6 manifestations. Combining insights from the free-energy principle with evolutionary

7 theorising in psychology, we argue that depression reflects an adaptive response to

8 perceived threats of aversive social outcomes (e.g., exclusion) that minimises the

9 likelihood of surprising interpersonal exchanges (i.e., those with unpredictable

10 outcomes). We suggest that psychopathology typically arises from ineffectual

11 attempts to alleviate interpersonal difficulties and/or hyper-reactive neurobiological

12 responses to social stress (i.e., uncertainty), which often stems from early experience

13 that social uncertainty is difficult to resolve.

14

15 Keywords: Active Inference; Evolutionary Systems Theory; Depression; Free-

16 Energy Principle; Major Depressive Disorder 


\section{An Evolutionary Systems Approach to Depression}

Why do we become depressed? Why are some of us particularly prone to depression? And how is this best managed? To answer these questions, we require an interdisciplinary approach that synthesises studies of the depressed brain with psychological research on its ecological, ontogenetic and biobehavioural correlates [1, 2]. To this end, we apply an integrative evolutionary systems theory (EST) of human brain function to explain depressed mood and its clinical manifestations. The EST in question rests on two uncontroversial assumptions. The first appeals to a consensus among cognitive scientists that the brain is a hierarchical, self-organising system sculpted by evolution [3-5]. This hierarchy ranges from lower-order, highly specialised neural subsystems responsible for sensory-motor processing; through to highly integrated cortical regions that develop more gradually and underlie the sophisticated, executive cognitive faculties unique to humans [see Box 1]. This calls for a theory of global brain function that explains how depression emerges from coordinated interactions within hierarchically integrated neuronal systems. The second assumption echoes dynamic systems approaches that situate the brain within the evolutionary dynamics of the brain-body-environment system [6-8]. According to this view, the neural mechanisms responsible for depression can only be understood by considering the broader context of human evolution, enculturation, development, embodiment and behaviour.

We aim to exemplify this approach by offering an interdisciplinary hypothesis of the depressed brain. Following the free-energy principle (FEP; see [5]), we first discuss how depressive disorders emerge from the functioning of, and disruptions to,

41 hierarchical neural dynamics that seek to minimise uncertainty. We then integrate this 
work with psychological research on the adaptive function of depression, along with the familial, developmental and psychobiological mechanisms that often underlie it.

44 We propose that our species-typical capacity for depressed mood can be explained as an evolved biobehavioural strategy that responds adaptively to adverse interpersonal conditions by minimising the likelihood of unpredictable social interactions. We discuss how our model builds on theories of clinical depression in the active inference literature, before turning to the hierarchical neural mechanics that underlie depressed mood and depressive disorder.

\section{Applying the Free-Energy Principle to Depression}

52 The FEP is a global theory of neural structure and function suggesting the brain can be seen as a "prediction machine" that attempts to maximise the evidence for a creature's model of the world by minimising an upper limit on surprise [i.e., freeenergy; see Box 2]. In line with predictive coding, the FEP describes the brain as a hierarchical generative model - a hierarchy of hypotheses about the world that enables a reduction of surprise by minimising discrepancies between incoming sensory inputs and top-down predictions [9]. Conditional expectations are thought to be encoded by deep pyramidal cells (i.e., representation units) at each level of the cortical hierarchy that convey predictions downward to suppress errors at the level

61 below, while prediction errors are encoded by superficial pyramidal cells (i.e., error

62 units) that convey errors forward to revise expectations at the level above [10]. This

63 allows us to minimise surprise by updating our internal models (i.e., perception).

64 Alternatively, we can selectively sample sensory data to ensure that our predictions

65 are self-fulfilling - by changing how we act upon the world to confirm our

66 expectations (i.e., active inference [11]). Thus, perception and action operate 
synergistically to minimise prediction errors and optimise our internal representations of the environment. A key corollary of this model is that our predictions are optimised by evolution, development and learning. Emphasis is placed on adaptive priors inherited expectations about the way our world unfolds that have been shaped by natural selection to guide action-perception cycles toward adaptive (i.e., unsurprising) states $[5,12]$.

To date, applications of the FEP to depressive disorders have chiefly concentrated on two processes, stemming from different levels of the cortical hierarchy. The first relates to limbic deficits in minimising prediction error. Barrett and colleagues suggest that depressive disorders arise from aberrant interoceptive predictions originating from abnormalities within the (limbic) agranular visceromotor cortex, which is central to emotional processing, energy regulation and allostatic responses to stress $[13,14]$. These abnormalities can arise from past exposure to sustained distress, and generate false (interoceptive) predictions about the body's upcoming autonomic, metabolic and immunological needs that chronically activate physiological stress responses (e.g., dysregulation of the hypothalamic-pituitary-adrenal (HPA) axis and pro-inflammatory states). Over time, the visceromotor systems try to minimise prediction error by producing sickness behaviours (e.g., negative affect and fatigue; also see [15]) that reduce energy expenditure and ultimately manifest in depression

87 [13]. Here, depression is seen as a disorder of allostasis, characterised by energy dysregulation and deficits in interoceptive inference; i.e., an insensitivity to prediction errors and/or a miscalibration of their precision - see glossary [14]. These deficits lead to a failure to update dysfunctional internal models (e.g., cognitive rigidity), 
91 perpetuating further metabolic inefficiencies and engendering the downward spiral 92 that typifies depressive illness.

94 The second class of models concentrates on impairments in top-down expectations of reward. Checkroud [16] has described the depressed brain as a hierarchical constellation of depressive beliefs, which impose a consistent negative bias in predictions that manifests in anhedonic features and the down-regulation of neural reward systems (e.g., dopaminergic and serotonergic dysfunction). In line with active

99 inference, these beliefs exacerbate depression by prompting the individual to actively 100 sample the environment to confirm negative predictions (e.g., learned helplessness).

101 Others have suggested that depressive disorders impair reward-approach behaviours 102 by causing a pathological underconfidence in one's predictions [17], or by distorting higher-order evaluations of the self (e.g., low self-worth), disrupting social behaviour

104 by overweighting the likelihood of aversive interactions [18]. Each of these proposals 105 echo models of reinforcement learning in computational psychiatry and evolutionary

106 biology suggesting that depression emerges from successive discrepancies between 107 actual and expected reward outcomes (i.e., prediction errors), entrenching (empirical) 108 prior beliefs that rewards are unlikely which inhibit reward-approach behaviours [19, $10920]$.

111 Taken together, the frameworks considered here suggest that depression entails 112 impairments in reward-approach systems emerging from two neurocognitive 113 processes - deficits in the predictive processing of sensory evidence; and prior beliefs 114 that negatively bias predictions. Although this perspective of depression as false 115 inference offers a cohesive, neurobiologically plausible account of the biobehavioural 
116 deficits observed in depressive illness, two important questions remain. First, by

117 concentrating on depressive disorders, the models above say little about our species-

118 typical capacity for depressed or low mood. The notable exception is a formal

119 (computational) scheme that defines emotional valence in terms of the rate of change

120 in free-energy over time, with positive and negative affect tracking a decrease and

121 increase in free-energy, respectively (see [17]). In this model, negative moods enable

122 an organism to respond adaptively to unexpected changes in the world by increasing

123 the (learning) rate of evidence accumulation - overweighing recent sensory inputs

124 over prior experiences to heighten sensitivity to environmental change, thereby

125 minimising prediction error [17]. However, this does not specifically address the

126 adaptive significance of depression per se. Second, the literature to date sheds little

127 light on the ecological conditions responsible for the positive selective pressure that

128 depression appears to have [see Box 3]. If depression instantiates an adaptive prior, it

129 should minimise surprise in response to specific environmental challenges that have

130 threatened our inclusive fitness (i.e., free-energy) over evolutionary time. Identifying

131 this adaptive function is arguably central to understanding why depression occurs. To

132 address these issues, we hope to build upon the active inference literature by

133 incorporating complementary insights drawn from an evolutionary systems approach

134 to psychology.

135

136 Insights from an Evolutionary Systems Approach to Psychology

137 In psychology, evolutionary systems models have typically focused on the dynamic

138 interplay between evolutionary and developmental processes (e.g., [8, 21-25]), an

139 approach that has been further extended to reconcile theoretical divisions between

140 major paradigms in the field (see [4]). According to this perspective, the embodied 
141 brain and behaviour emerge from selection acting on dynamic interactions between

142 the levels of causation identified by Tinbergen - adaptation, phylogeny, ontogeny,

143 and mechanism [26]. This causal hierarchy is arguably recapitulated by paradigms in

144 psychology, which concentrate differentially on four overlapping levels of

145 explanation - ultimate hypotheses for adaptive, species-typical characteristics (i.e.,

146 evolutionary psychology); epigenetic explanations for intergenerational, between-

147 group differences (i.e., evolutionary developmental biology and psychology);

148 dynamical explanations for individual similarities and differences (i.e., developmental

149 psychology); and mechanistic explanations for real-time phenomena (i.e.,

150 psychological subdisciplines such as cognitive, biological, personality, social and

151 clinical psychology) [4]. Central to EST is the need to explore how these causal levels

152 interact - evolutionary influences on neural structure and function constrain

153 individual development and learning, while effects at these more proximate levels can

154 shape the evolution of the brain $[3,27]$. To explain depression then, we require a

155 multi-level hypothesis that synthesises diverse fields of psychological inquiry to

156 explain both why it is adaptive, along with how it emerges from intergenerational,

157 developmental and real-time mechanisms.

159 Although there are various Darwinian models of depression [28], a theme common to

160 many of these is that low mood reflects an adaptive biobehavioural strategy that

161 conserves or reallocates energy and resources in unpropitious social environments [29,

$16230]$. According to this view, depressed mood states are elicited by aversive

163 interpersonal outcomes (e.g., exclusion, defeat, or loss) that indicate a critical loss of

164 control over social relationships that were critical to ancestral fitness [31]. A model

165 that incorporates influential theories in this area and shows promising conceptual 
parallels with the FEP is the social risk hypothesis (technically, risk corresponds to uncertainty and uncertainty is expected surprise or free energy). This maintains that depressed mood reflects an adaptive, risk-averse approach to social interaction that reduces the likelihood of further aversive outcomes by: (1) increasing our cognitive sensitivity to (sensory) cues of social risk; (2) reducing our (behavioural) propensity

171 for taking social risks; and (3) initiating signalling behaviours that elicit support and defuse conflict [32].

174 The idea that depression reflects an evolved response to adverse social conditions 175 concords with evidence that extends across Tinbergen's remaining levels of inquiry.

176 The intergenerational transmission of susceptibility to depressive disorders due to 177 deleterious social environments is widely documented [33, 34], with studies involving rodents, primates, and humans showing that exposure to social stressors (e.g., low maternal care and social defeat) produces potentially heritable epigenetic changes that 180 confer risk for disorder by heightening stress reactivity [35, 36]. Ontogenetically, 181 exposure to early social stress (e.g., parental loss, abuse, or neglect) is a strong 182 predictor of depressive vulnerability [37], and is thought to heighten susceptibility to 183 disorder by leading to hyperactivity of the HPA axis [38, 39] and up-regulating pro184 inflammatory immune responses [40]. Behavioural and neuroimaging studies further 185 suggest that the risk of depressive onset rises markedly in adolescence because of an 186 increased sensitivity to social threats in this period [41, 42]. Finally, research across 187 the sub-disciplines highlights an intimate connection between depression and the 188 social world (see [43]). For example, the precipitants of depression are typically 189 interpersonal in nature [44]; social support and belonging are key protective factors 190 [45]; and typical correlates of depression clearly exemplify negative self-other 
191 relations (e.g., low self-esteem [46]). Consistent with the social risk hypothesis, there 192 are also multiple lines of evidence to suggest that low mood is associated with 193 biobehavioural changes that facilitate adaptive responses to social stress. Depressive 194 cognition is characterised by a specific, attentional bias towards socially-threatening 195 stimuli [47] and increased rumination about interpersonal problems [48], while 196 normative depressed states have been shown to increase the accuracy of social 197 inferences (e.g., depressive realism [49]) and improve social problem-solving [50]. 198 Furthermore, many features of depression - such as anhedonia, a negative thinking 199 bias and social withdrawal - reduce exposure to social risks by inhibiting reward200 approach behaviours [51], while the signalling behaviours associated with depression 201 (e.g., reassurance-seeking and submissive behaviours) explicitly attempt to elicit 202 support and defuse potential conflict [52-54]. Notably, other studies have provided 203 direct empirical support for the social risk hypothesis itself (Badcock \& Allen, 2003; 204 Badcock \& Allen, 2007; Dunn, Whelton \& Sharpe, 2012; Girard, Cohn, Mahoor, 205 Mavadati, \& Rosenwald, 2013). In light of such work, we suggest that the human capacity for depressed mood can be explained in terms of a risk-averse adaptive prior that minimises uncertainty in the 209 social world when sensory cues indicate both a high degree of socio-environmental 210 volatility (i.e., unpredictability) and an increased probability of aversive interpersonal 211 outcomes (e.g., rejection, defeat or loss) (see Figure 1). This depressive response 212 instantiates a "better safe than sorry" strategy that minimises the likelihood of 213 unpredictable social interactions by causing adaptive changes in cognition (e.g., 214 hypersensitivity to aversive social stimuli, a negative thinking bias and deficits in 215 responses to reward) and action (e.g., risk-averse behaviours such as social 
216 withdrawal). Epigenetic and ontogenetic mechanisms arguably support this function

217 by sensitising the individual to socio-environmental volatility when developmental

218 insults indicate that the probability of aversive social interactions is high, producing

219 hyper-reactive stress response systems that heighten risk for disorder by increasing

220 the precision of social prediction errors and prompting exaggerated, pathological

221 responses to interpersonal stressors.

222

223 Notably, the exacerbation of normative depressed states into severe, dysfunctional

224 forms is also likely when depressive changes fail to alleviate social stress, creating a

225 self-perpetuating cycle arising from heightened and prolonged arousal of ineffectual 226 attempts to reduce socio-environmental volatility [32]. This, in turn, is likely to

227 chronically activate neurophysiological stress responses and leads to debilitating

228 sickness behaviours $[13,14]$. As discussed above, previous applications of the FEP

229 suggest that this depressive spiral is engendered by a positive feedback loop between

230 two neurocognitive mechanisms - the increased precision of social prediction errors,

231 coupled with a negative bias affecting social predictions. Following active inference,

232 this is likely to engender ongoing depressive behaviours that seek to confirm negative

233 biases, creating a self-fulfilling prophecy (i.e., high predictability) born from mutually

234 reinforcing patterns of cognition and behaviour [16]. Here, depressed can be

235 interpreted as a maladaptive pattern of dysregulated defences - if this depressive

236 response is effective, an individual either escapes or avoids the social stressor or

237 adapts to it; if the defence fails, the individual is at risk of entering a self-perpetuating

238 dysregulated state, which falls beyond the normal range of adaptive functioning

239 (Allen \& Badcock, 2003; Gilbert, 2001). Nevertheless, it should also be recognised

240 that clinical manifestations of depression can result from asocial causes that produce 
241 neurobiological abnormalities typically associated with dysregulated mood (e.g., pro-

242 inflammatory immune responses induced by illness and medications; 40).

\section{The Depressed Brain}

245 It is widely accepted that depression emerges from bidirectional interactions between

246 hierarchically organised neural regions. Most of the theoretical work in this area

247 concentrates on two general brain systems that work in concert - a ventral affective

248 system, including subcortical regions such as the amygdala and ventral striatum; and

249 the prefrontal cortex (PFC), which modulates the reactions of the ventral affective

250 system [1]. These systems are composed of subcortical neural circuits responsible for

251 the unconscious processing of affective and social stimuli on the one hand; and on the

252 other, executive networks that regulate affective states, with medial prefrontal regions

253 playing a particularly important role in modulating visceral and behavioural responses

254 in order to adapt them to the external milieu [1].

256 More particularly, evidence gleaned from neuroimaging and animal studies suggests

257 that depression involves dysfunction of the "extended visceromotor network", in

258 which the medial PFC regulates affective states by modulating visceromotor output

259 via connections with the amygdala, ventral striatum, hypothalamus, and other

260 subcortical regions [55]. Brain regions across this network regulate motivation (e.g.,

261 anhedonia and dopaminergic function) and neurobiological responses to stress, and

262 play a central role in social threat and reward processing [39, 41, 56, 57].

263 Neurodevelopmental changes in these regions throughout adolescence are also

264 thought to heighten vulnerability to disorder by increasing sensitivity to rapidly

265 changing social contexts in this period (see Box 4). Collectively, such findings fit well 
266 with our proposal that depression often stems from the need to adapt to complex

267 social contexts, and manifests through the bidirectional interplay of hierarchical

268 neuronal processes.

270 Specifically, we speculate that the extended visceromotor system responds to

271 volatility in the social environment by increasing the precision of social prediction

272 errors, initiating changes in neuronally encoded expectations that increase attention to

273 social cues and motivate risk-averse behaviours (e.g., social withdrawal). This

274 heightened sensitivity to somatic and affective cues leads, in turn, to further avoidance

275 of interpersonal stressors. The depressive response is adaptive when changes in mood

276 state and behaviour reduce uncertainty in the face of socio-environmental change, and

277 lead to re-engagement with that environment when volatility abates (which should, at

278 least in part, be brought about by depressive behaviours; see [32]). However,

279 following the active inference literature, we suggest that the depressive response

280 becomes maladaptive when there are (neuromodulatory) failures of "precision

281 engineered" visceromotor inference - produced, for instance, by sustained social

282 distress - leading to illness behaviours which fail to respond to improvements in

283 interpersonal contexts and can often exacerbate socio-environmental stress [13, 14].

284 Neurodevelopmentally, the PFC can also potentiate vulnerability to depression by

285 underwriting the formation of distal goals that, when frustrated by rejection or failure,

286 can lead to depression by suppressing the brain's reward system [58] and the

287 confidence in (or precision of) our beliefs about behaviour [59], thereby inhibiting 288 goal-directed behaviours. 
290 Ultimately, our basic claim is that depression can be viewed as an adaptive faculty

291 that underwrites emotional allostasis in an increasingly prosocial and volatile world.

292 Physiologically, this faculty increases sensitivity to interpersonal, affiliative and

293 interoceptive cues. Clearly, sensitisation to stressful exteroceptive and interoceptive

294 cues also has to be predicted by the hierarchical brain, which implicates the functional

295 neuroanatomy described above. Under active inference, sensitivity to stress-related

296 cues corresponds to their precision [13, 60], implicating neuromodulatory systems

297 associated with reward, action selection and interoceptive inference [61-63].

298 Crucially, in order to act it is also necessary to attenuate the precision afforded to the

299 sensory consequences of action (i.e., we have to ignore the fact that we are not

300 currently acting). This means that an adaptive depressive response suspends sensory

301 attenuation - and action - so that we can attend to interpersonal prediction errors and

302 revise our (posterior) beliefs about our relationships with others, via perceptual

303 inference and learning. Sensory attenuation can be regarded as the complement of

304 sensory attention; i.e., attenuating or augmenting the gain (precision) afforded sensory

305 prediction errors to ignore or select sensory information, respectively. According to

306 this scheme, maladaptive forms of depression reflect a pervasive, self-maintaining

307 failure of sensory attenuation, leading to ruminations, false inference and a

308 concomitant inability to act and test these false beliefs.

310 Interestingly, exactly the same conclusions (namely, a failure of sensory attenuation)

311 have been drawn for a range of neuropsychiatric disorders, ranging from autism [64]

312 to schizophrenia [65]. One could ask what is specific about this mechanism in

313 depression, and respond by referring to the particular (interoceptive and affiliative)

314 modalities affected. However, perhaps the more intriguing implication is that the 
comorbidity of depression and other disorders might arise from a common

pathophysiological mechanism, which can be explained in terms of false inference.

\section{Concluding Remarks}

319 In this opinion piece, we have endeavoured to contribute to the active inference

320 literature on mood disorder by suggesting that normative levels of depressed mood

321 instantiate an adaptive prior that minimises the likelihood of surprising interpersonal

322 interactions when faced with threats of aversive social outcomes that typically

323 compromised ancestral fitness. By extending beyond previous applications of the FEP

324 to emphasise both the adaptive function of low mood and the causal role of the social

325 ecology, we believe our model demonstrates the heuristic benefits of combining

326 active inference with insights in psychology to improve our understanding of

327 depressed mood and mood disorder. It also motivates new questions for research,

328 calling for greater integration between neuroscientific and psychological approaches

329 to explore the ways in which the neural mechanisms that underpin depression relate to

330 behaviour, development and the social world [see Outstanding Questions]. In

331 particular, the idea that depression can emerge from the need to navigate social risks

332 stands to inform theory-driven approaches in computational psychiatry, which

333 improve our understanding, prediction and treatment of mental illness by using

334 simulations and mathematical models to capture complex interactions across multiple

335 causal levels $[19,66]$.

337 That said, we do not wish to imply that depression is solely attributable to social 338 causes. In evolutionary psychology, for instance, the distinction between social and 339 non-social depressive responses is widely recognised [Durisko 2015; Gilbert 2006], 
and as we have noted, depression can also arise from depressogenic neuroanatomical

341 abnormalities produced by influences other than unfavourable social conditions.

342 Nevertheless, our model adds to the active inference literature by emphasising the

343 importance of the social environment in explaining the aetiology and phenomenology

344 of depression. This underscores the need to develop (computational) diagnostic tools

345 that are capable of distinguishing between social and non-social forms in order to 346 inform treatment decisions.

348 In closing, our model also promotes clear avenues for intervention. To date,

349 proponents of the FEP have advocated treatments that directly target dysregulated

350 neural systems, such as psychopharmacological agents that act upon the

351 neurotransmitter systems that encode precision or uncertainty (e.g., serotonin and

352 dopamine [16]). They have also recommended the use of cognitive behavioural

353 therapies to disrupt the spiral of self-defeating actions typical of depression [16], or to

354 construct new prediction signals that modify the gain on prediction errors via the

355 salience network [14]. Our own model adds to this work by emphasising the need to

356 facilitate adaptive responses to social stress. This could well explain the efficacy of

357 interpersonal psychotherapy as a treatment for major depressive disorder [67], and

358 highlights the value of prevention and early intervention efforts that reduce

359 vulnerability by targeting modifiable risk factors in the social environment. Given the

360 heterogeneous nature of depression, we also recommend the development of

361 (computational) diagnostic tools capable of distinguishing between social and non-

362 social forms in order to inform treatment decisions. Finally, simply having a positive

363 and principled framework within which to understand depression - and the rationale

364 for therapeutic interventions - is likely to be helpful for those seeking treatment. Our 
365 synthesis can be used to help clients understand why they have depression, and to

366 explain why, for example, it might be useful to combine interpersonal psychotherapy

367 with antidepressants.

368

369 Acknowledgements: We would like to thank Lucy Morrish, Jakob Hohwy, Alex

370 Fornito, Rebecca Schwarzlose and three anonymous reviewers for their valuable

371 contributions. Karl Friston is funded by the Wellcome Trust; Christopher Davey and

372 Sarah Whittle are both funded by the National Health and Medical Research Council

373 of Australia (NHMRC). 
375 Active inference: A corollary of the free-energy principle, which states that we

376 minimise surprise (i.e., prediction errors) by changing our predictions (i.e.,

377 perception) or by acting upon the world to elicit sensations that conform to

378 predictions (i.e., action).

379 Adaptive prior: A prior endowed by evolution to underwrite adaptive fitness.

380 Association cortex: Regions of the cerebral cortex that are not primary sensory or

381 motor projection areas, including the prefrontal cortex, and extensive parts of the

382 temporal, parietal and occipital cortices..

383 Empirical priors: Priors found in hierarchical models that can be learned or inferred

384 under priors from the level above.

385 Entropy: The uncertainty or average surprise associated with outcomes sampled from

386 a probability distribution. A distribution with low entropy means, on average, that the 387 outcome is relatively predictable.

388 Evolutionary systems theory: A multidisciplinary paradigm that explains dynamic,

389 evolving systems in terms of co-action between self-organisation and general 390 selection (e.g., natural selection) over time. This produces complex adaptive systems,

391 like the brain, that adapt to the environment through an autonomous process of

392 selection that recruits the outcomes of locally interacting components within that

393 system to select a subset of those components for replication or enhancement.

394 Free-energy principle: A generalisation of predictive coding that asserts that

395 organisms actively minimise an upper bound on surprise (i.e., free energy), which,

396 under simplifying assumptions, translates to (precision weighted) prediction error. 
397 Generative Model: A probabilistic mapping from hidden causes in the environment to observed consequences (sensory data), typically specified in terms of the likelihood of observing some data (given their causes) and priors (on these causes).

400 Interoception: The perception and integration of autonomic, hormonal, visceral and 401 immunological (bodily) signals.

402 Precision: The inverse variance, volatility, or reliability of a signal. In predictive 403 coding, prediction errors are weighted by precisions that determine the relative 404 influence of bottom-up (error) and top-down (representation) signals (e.g., a high 405 precision on error signals corresponds to low confidence in top-down beliefs).

406 Dynamic precision weighting is mediated by neuromodulation and underwrites 407 psychological processes such as attentional selection and sensory attenuation. 408 Predictive coding: A processing scheme for inferring the likely causes of sensory 409 data by minimising prediction error. Typically, this entails a hierarchical generative 410 model (e.g., the brain) in which top-down signals convey predictions and bottom-up

411 signals convey (precision weighted) prediction errors.

412 Prior: The probability distribution or density on the causes of data that encode beliefs 413 about those causes prior to observing the data.

414 Surprise: The negative log probability of sensory experiences encountered by an 415 agent. Also known as surprisal or self-information.

416 Visceromotor cortex: Agranular (limbic) regions of isocortex and allocortex that 417 regulate the hormonal, immune and autonomic nervous systems, including the 418 cingulate cortex, the posterior ventral medial prefrontal cortex, the posterior 419 orbitofrontal cortex and ventral portions of the anterior insula. 
421 In psychology, it has long been recognised that the brain entails a hierarchical

422 structure ranging from highly specialised sensorimotor systems at its lowest levels

423 through to developmentally flexible, highly integrated systems responsible for higher-

424 order executive functions [3, 4]. A hierarchical neural architecture is also emphasised

425 by predictive coding approaches in neuroscience, which explore how the brain

426 minimises prediction error via recurrent message-passing between cortical levels [9,

$42768,69]$. More recently, imaging studies in network neuroscience have provided direct

428 evidence that the brain exhibits a multiscale hierarchical organisation, with a given

429 node (e.g., network, module or sub-module) itself comprising a network of smaller

430 interacting nodes at a lower level $[68,70]$ (see Figure I).

432 Comparative work suggests that a hierarchical architecture is a hallmark of the

433 mammalian brain, progressing from highly segregated sensorimotor hierarchies

434 common to all mammals through to the cortical association areas that confer the

435 adaptive advantage of heightened cognitive control among primates [71, 72]. Again,

436 this structure is thought to exemplify the complementary relationship between

437 evolution and development - selection has canalised early sensorimotor regions that

438 serve as neurodevelopmental anchors, allowing for the progressive, activity-

439 dependent self-organisation of widely distributed association networks that lie furthest

440 from sensory patterning centres $[71,73]$. This neuroplasticity enhances adaptability

441 by producing higher-order, "domain-general" faculties that are able to respond

442 flexibly to rapidly changing environments [6, 73]. 
444 It is now broadly accepted that a hierarchical neural structure is favoured by selection.

445 It enhances evolvability because deleterious changes to a single component of the

446 system are unlikely to affect the system itself, and it allows adaptive novelties to

447 emerge without disrupting global functioning [70]. Computer simulations of evolving

448 networks have also shown that selection favours a hierarchical organisation because it

449 conserves the (spatial, processing and metabolic) cost of neural connections; improves

450 problem-solving by recursively combining solutions to sub-problems; and adapts

451 more rapidly to new environments than non-hierarchical structures [74]. Finally, the

452 hierarchical brain is thought to promote "self-organised criticality". This is a

453 dynamical state poised between completely ordered, stable cycles of activity and

454 highly complex, chaotic ones that optimises evolvability because it allows small

455 extrinsic changes to elicit large intrinsic reorganisations. The hierarchical segregation

456 of neural networks into distributed neighbourhoods has been found to stretch the

457 parameter range for self-organised criticality by allowing subcritical and supercritical

458 dynamics to co-exist simultaneously [75]. Since systems at criticality have optimal

459 information-processing capacities, a structure that extends this critical region is likely

460 to be naturally selected [76]. 
462 The FEP seeks to explain how biological systems maintain their integrity by

463 occupying a constricted number of states [5]. It suggests that all organisms actively

464 reduce the entropy (i.e. disorder or dispersion) of their sensory and physical states by

465 minimising free-energy. Borrowed from statistical thermodynamics and machine

466 learning, free-energy is an information theory quantity which limits (by being greater

467 than) the entropy of a brain's sensations or sensory samples from the environment. In

468 this context, entropy (the mathematical description of uncertainty) refers to the (long-

469 term) average of surprise: a statistical concept referring to the negative log probability

470 of sensory samples encountered by an agent. This probability is also known as

471 (Bayesian) model evidence.

These principles have important implications for understanding how biotic agents

work. Because the repertoire of states an organism occupies is limited, the probability of these states has low entropy (i.e., surprise). Thus, an organism's distal imperative of maintaining functional states within physiological bounds (i.e., homeostasis) translates into a proximal avoidance of surprise [5]. Surprise itself cannot be evaluated; however, biological systems can minimise surprise vicariously by minimising their free-energy - which roughly translates to prediction error, weighted by its precision.

482 The FEP appeals to predictive coding by characterising the brain as a hierarchical 483 inference machine that minimises prediction error by seeking to match incoming 484 sensory inputs with top-down predictions [see Figure II]. This occurs in two ways.

485 First, we can improve our predictions by altering internal states (i.e., perception).

486 Second, we can act upon the world to confirm our predictions (i.e., action). Thus, 
action and perception operate synergistically to optimise an organism's model of the environment. Crucially, to minimise free-energy, the precision of prediction errors also has to be predicted, invoking notions of attentional gain (psychologically) and neuromodulation (physiologically).

492 The FEP also applies to the morphology, development and evolution of the brain. It 493 suggests that instead of just containing a model of the world, the brain is a model of 494 the world - a physical transcription of causal regularities in the environment that is 495 optimised by evolution. This model instantiates genetically specified (empirical) prior 496 beliefs that have minimised free-energy (i.e., maximised model evidence) over 497 evolutionary time by ensuring an organism seeks out a small number of unsurprising 498 states that are consistent with its phenotype and environment. In other words, natural 499 selection is nature's way of performing Bayesian model selection to minimise the 500 (variational) free energy of phenotypes (i.e., generative models). 
Box 3: The Adaptive Significance of Depression 


\section{Box 4: The Adolescent Brain and Risk for Depression}

504 The brain undergoes significant maturation in adolescence, involving processes that

505 begin with puberty and continue until a young person is in their mid-to-late twenties

506 [77]. Over this period, there is a progressive increase in white matter, alongside

507 synaptic pruning and grey matter loss, which have the effect of delineating more

508 clearly defined large-scale brain networks [78]. Subcortical regions, including the

509 primary components of the reward system, undergo more rapid maturation [79], while

510 the most prolonged development is in association cortex, including prefrontal regions

511 that are implicated in social processing $[78,80]$.

512

513 It is now widely accepted that the functional and structural changes that accompany

514 adolescence reflect a particularly sensitive period for adapting to the social world.

515 Brain imaging studies show that adolescence is typified by significant alterations in

516 social and affective processing systems, which are thought to increase risk for mood

517 disorder by heightening sensitivity to social threats in this period [41, 80-82].

518 Coincident with these neurodevelopmental processes, there are also substantial

519 changes in the adolescent social environment. Peer relationships become increasingly

520 important, hierarchical and complex, and there is significant socio-environmental

521 volatility - friendships change frequently, and romantic relationships are typically

522 short-lived [83].

523

524 It is unsurprising, then, that the period from adolescence to early adulthood is a peak

525 time for the onset of depression [42]. During adolescence, sources of social

526 uncertainty are frequently encountered. Maturation of subcortical regions, along with

527 marked hormonal changes [82, 84], increase sensitivity to affective and self-relevant 
528 social cues. Moreover, prefrontal cortical development leads, on the one hand, to

529 improved regulation of affective processes, but on the other, heightens sensitivity to

530 the nuance and complexity of interpersonal relationships [58]. For this reason,

531 increased vulnerability to depression starts in puberty but is maintained well beyond 532 adolescence.

533 


\section{References}

535

536

537

538

539

540

541

542

543

544

545

546

547

548

549

550

551

552

553

554

555

556

557

558

1. Pfeifer, J.H. and Allen, N.B. (2012) Arrested development? Reconsidering dualsystems models of brain function in adolescence and disorders. Trends Cognit. Sci. $16,322-329$.

2. Pfeifer, J.H. and Allen, N.B. (2016) The audacity of specificity: Moving adolescent developmental neuroscience towards more powerful scientific paradigms and translatable models. Dev. Cogn. Neurosci. 17, 131-137.

3. Badcock, P.B. (2012) Evolutionary systems theory: A unifying meta-theory of psychological science. Rev. Gen. Psychol. 16, 10.

4. Friston, K. (2010) The free-energy principle: A unified brain theory? Nat. Rev. Neurosci. 11, 127-138.

5. Badcock, P. et al. (submitted) The hierarchically mechanistic mind: An evolutionary systems theory of the brain and behaviour.

6. Anderson, M.L. (2014) After Phrenology, MIT Press.

7. Clark, A. (2015) Surfing Uncertainty: Prediction, Action, and the Embodied Mind, Oxford University Press.

8. Lickliter, R. and Honeycutt, H. (2003) Developmental dynamics: toward a biologically plausible evolutionary psychology. Psychol. Bull. 129, 819-835.

9. Clark, A. (2013) Whatever next? Predictive brains, situated agents, and the future of cognitive science. Behav. Brain. Sci. 36, 181-204.

10. Bastos, A.M. et al. (2012) Canonical microcircuits for predictive coding. Neuron $76,695-711$

11. Friston, K.J. et al. (2010) Action and behavior: A free-energy formulation. Biol. Cybern. 102, 227-260.

12. Friston, K. (2013) Life as we know it. J. R. Soc. Interface 10, 20130475. 
559 13. Barrett, L.F. and Simmons, W.K. (2015) Interoceptive predictions in the brain.

$560 \quad$ Nat. Rev. Neurosci. 16, 419-429.

561 14. Barrett, L.F. et al. (2016) An active inference theory of allostasis and

562 interoception in depression. Philos. Trans. R. Soc. Lond. B Biol. Sci. 371,

56320160011.

564 15. Seth, A.K. and Friston, K.J. (2016) Active interoceptive inference and the

565 emotional brain. Philos. Trans. R. Soc. Lond. B Biol. Sci. 371, 20160007.

566 16. Chekroud, A.M. (2015) Unifying treatments for depression: An application of the

$567 \quad$ Free Energy Principle. Front Psychol 6, 153.

568 17. Joffily, M. and Coricelli, G. (2013) Emotional valence and the free-energy

569 principle. PLoS Comput. Biol. 9, e1003094.

570 18. Moutoussis, M. et al. (2014) Bayesian inferences about the self (and others): A

571 review. Conscious. Cogn. 25, 67-76.

572 19. Adams, R.A. et al. (2016) Computational psychiatry: Towards a mathematically

573 informed understanding of mental illness. J. Neurol. Neurosurg. Psychiatry 87, $574 \quad 53-63$.

575 20. Nettle, D. and Bateson, M. (2012) The evolutionary origins of mood and its 576 disorders. Current Biology 22, R712-R721.

577 21. Caporael, L.R. (2001) Evolutionary psychology: Toward a unifying theory and a 578 hybrid science. Annu. Rev. Psychol. 52, 607-628.

579 22. Geary, D.C. and Bjorklund, D.F. (2000) Evolutionary developmental psychology.

$580 \quad$ Child Dev. 71, 57-65.

581 23. Kenrick, D.T. et al. (2002) Dynamical evolutionary psychology: Mapping the 582 domains of the new interactionist paradigm. Pers. Soc. Psychol. Rev. 6, 347-356. 
24. Ploeger, A. et al. (2008) Is evolutionary psychology a metatheory for psychology? A discussion of four major issues in psychology from an evolutionary developmental perspective. Psychol. Inq. 19, 1-18.

25. Frankenhuis, W.E. et al. (2013) Bridging developmental systems theory and evolutionary psychology using dynamic optimization. Dev. Sci. 16, 584-598.

26. Tinbergen, N. (1963) On aims and methods of ethology. Z. Tierpsychol. 20, 410433.

27. Marshall, P.J. (2013) Coping with complexity: Developmental systems and multilevel analyses in developmental psychopathology. Dev. Psychopathol. 25, $1311-1324$.

28. Durisko, Z. et al. (2015) An adaptationist perspective on the etiology of depression. J. Affect. Disord. 172, 315-323.

29. Beck, A.T. and Bredemeier, K. (2016) A unified model of depression integrating clinical, cognitive, biological, and evolutionary perspectives. Clin. Psychol. Sci. 4, 596-619.

30. Trimmer, P.C. et al. (2015) Adaptive learning can result in a failure to profit from good conditions: Implications for understanding depression. Evol. Med. Public Health 2015, 123-135.

31. Gilbert, P. (2006) Evolution and depression: Issues and implications. Psychol. Med. 36, 287-297.

32. Allen, N.B. and Badcock, P.B. (2003) The social risk hypothesis of depressed mood: Evolutionary, psychosocial, and neurobiological perspectives. Psychol. Bull. 129, 887-913.

33. Vialou, V. et al. (2013) Epigenetic mechanisms of depression and antidepressants action. Annu. Rev. Pharmacol. Toxicol. 53, 59-87. 
34. Weissman, M.M. et al. (2005) Families at high and low risk for depression: A 3generation study. Arch. Gen. Psychiatry 62, 29-36.

35. Sun, H. et al. (2013) Epigenetics of the depressed brain: Role of histone acetylation and methylation. Neuropsychopharmacol. 38, 124-137.

36. Meaney, M.J. (2001) Maternal care, gene expression, and the transmission of individual differences in stress reactivity across generations. Annu. Rev. Neurosci. $24,1161-1192$.

37. Heim, C. and Binder, E.B. (2012) Current research trends in early life stress and depression: Review of human studies on sensitive periods, gene-environment interactions, and epigenetics. Exp. Neurol. 233, 102-111.

38. Gold, P. (2015) The organization of the stress system and its dysregulation in depressive illness. Mol. Psychiatry 20, 32-47.

39. De Raedt, R. and Koster, E.H. (2010) Understanding vulnerability for depression from a cognitive neuroscience perspective: A reappraisal of attentional factors and a new conceptual framework. Cogn. Affect. Behav. Neurosci. 10, 50-70.

40. Slavich, G.M. and Irwin, M.R. (2014) From stress to inflammation and major depressive disorder: A social signal transduction theory of depression. Psychol. Bull. 140, 774.

41. Silk, J.S. et al. (2012) Why do anxious children become depressed teenagers? The role of social evaluative threat and reward processing. Psychol. Med. 42, 20952107.

42. Andersen, S.L. and Teicher, M.H. (2008) Stress, sensitive periods and maturational events in adolescent depression. Trends Neurosci. 31, 183-191.

43. Gotlib, I.H. and Hammen, C. (2014) Handbook of Depression (3rd edn.), Guilford Press. 
44. Vrshek-Schallhorn, S. et al. (2014) Refining the candidate environment interpersonal stress, the serotonin transporter polymorphism, and geneenvironment interactions in major depression. Clin. Psychol. Sci. 2, 235-248.

45. Cohen, S. and Wills, T.A. (1985) Stress, social support, and the buffering hypothesis. Psychol. Bull. 98, 310-357.

46. Hawkley, L.C. and Capitanio, J.P. (2015) Perceived social isolation, evolutionary fitness and health outcomes: A lifespan approach. Philos. Trans. R. Soc. Lond. B Biol. Sci. 370, 20140114.

47. Leppänen, J.M. (2006) Emotional information processing in mood disorders: A review of behavioral and neuroimaging findings. Curr. Opin. Psychiatry 19, 34-39.

48. Hankin, B.L. et al. (2010) Corumination, interpersonal stress generation, and internalizing symptoms: Accumulating effects and transactional influences in a multiwave study of adolescents. Dev. Psychopathol. 22, 217-235.

49. Moore, M.T. and Fresco, D.M. (2012) Depressive realism: A meta-analytic review. Clin. Psychol. Rev. 32, 496-509.

50. Forgas, J.P. (2016) Can sadness be good for you? Aust. Psychol. DOI: 10.1111/ap.12230

51. Pizzagalli, D.A. (2014) Depression, stress, and anhedonia: Toward a synthesis and integrated model. Annu. Rev. Clin. Psychol. 10, 393.

52. Hagen, E.H. (2011) Evolutionary theories of depression: A critical review. Can. J. Psychiatry 56, 716-726.

53. Hames, J.L. et al. (2013) Interpersonal processes in depression. Annu. Rev. Clin. Psychol. 9, 355-377.

54. Sloman, L. and Gilbert, P. (2000) Subordination and Defeat: An Evolutionary approach to Mood Disorders and Their Therapy, Routledge. 
55. Price, J.L. and Drevets, W.C. (2012) Neural circuits underlying the pathophysiology of mood disorders. Trends Cogn. Sci. 16, 61-71.

56. Kupferberg, A. et al. (2016) Social functioning in major depressive disorder. Neurosci. Biobehav. Rev. 69, 313-332.

57. Rushworth, M.F. et al. (2013) Are there specialized circuits for social cognition and are they unique to humans? Curr. Opin. Neurobiol. 23, 436-442.

58. Davey, C.G. et al. (2008) The emergence of depression in adolescence: Development of the prefrontal cortex and the representation of reward. Neurosci. Biobehav. Rev. 32, 1-19.

59. Friston, K. et al. (2015) Active inference and epistemic value. Cogn. Neurosci., 128.

60. Friston, K. et al. (2014) The anatomy of choice: Dopamine and decision-making. Philos. Trans. R. Soc. Lond. B Biol. Sci. 369, 20130481.

61. Paulus, M.P. and Stein, M.B. (2006) An insular view of anxiety. Biol. Psychiatry 60, 383-387.

62. Pezzulo, G. et al. (2015) Active Inference, homeostatic regulation and adaptive behavioural control. Prog. Neurobiol. 134, 17-35.

63. Waselus, M. et al. (2011) Collateralized dorsal raphe nucleus projections: A mechanism for the integration of diverse functions during stress. J. Chem. Neuroanat. 41, 266-280.

64. Pellicano, E. and Burr, D. (2012) When the world becomes 'too real': A Bayesian explanation of autistic perception. Trends Cogn. Sci. 16, 504-510.

65. Fletcher, P.C. and Frith, C.D. (2009) Perceiving is believing: A Bayesian approach to explaining the positive symptoms of schizophrenia. Nat. Rev. Neurosci. 10, 48-58. 
683

684

685

686

687

688

689

690

691

692

693

694

695

696

697

698

699

700

701

702

703

704

705

706

66. Friston, K.J. et al. (2014) Computational psychiatry: The brain as a phantastic organ. Lancet Psychiatry 1, 148-158.

67. Cuijpers, P. et al. (2011) Interpersonal psychotherapy for depression: A metaanalysis. Am. J. Psychiatry 168, 581-592.

68. Park, H.-J. and Friston, K. (2013) Structural and functional brain networks: from connections to cognition. Sci. 342, 1238411.

69. Hohwy, J. (2013) The Predictive Mind, Oxford University Press.

70. Sporns, O. and Betzel, R.F. (2016) Modular brain networks. Annu. Rev. Psychol. 67, 613-640.

71. Buckner, R.L. and Krienen, F.M. (2013) The evolution of distributed association networks in the human brain. Trends Cogn. Sci. 17, 648-665.

72. Finlay, B.L. and Uchiyama, R. (2015) Developmental mechanisms channeling cortical evolution. Trends Neurosci. 38, 69-76.

73. Anderson, M.L. and Finlay, B.L. (2014) Allocating structure to function: The strong links between neuroplasticity and natural selection. Front. Hum. Neurosci. $7,918$.

74. Mengistu, H. et al. (2016) The evolutionary origins of hierarchy. PLOS Comput. Biol. 12, e1004829.

75. Hilgetag, C.C. and Hütt, M.-T. (2014) Hierarchical modular brain connectivity is a stretch for criticality. Trends Cogn. Sci. 18, 114-115.

76. Hesse, J. and Gross, T. (2014) Self-organized criticality as a fundamental property of neural systems. Front. Syst. Neurosci. 8, 46-59.

77. Sowell, E.R. et al. (2003) Mapping cortical change across the human life span. Nat. Neurosci. 6, 309-315. 
78. Blakemore, S.-J. (2012) Imaging brain development: the adolescent brain.

Neuroimage 61, 397-406.

79. Spear, L.P. (2000) The adolescent brain and age-related behavioral manifestations. Neurosci. Biobehav. Rev. 24, 417-463.

80. Fuhrmann, D. et al. (2015) Adolescence as a sensitive period of brain development. Trends Cogn. Sci. 19, 558-566.

81. Blakemore, S.-J. and Mills, K.L. (2014) Is adolescence a sensitive period for sociocultural processing? Annu. Rev. Psychol. 65, 187-207.

82. Crone, E.A. and Dahl, R.E. (2012) Understanding adolescence as a period of social-affective engagement and goal flexibility. Nat. Rev. Neurosci. 13, 636-650.

83. Brown, B.B. and Larson, J. (2009) Peer relationships in adolescence. In Handbook of Adolescent Psychology (3rd edn) (Lerner, R.M. and Steinberg, L. eds), pp. 74103, John Wiley \& Sons.

84. Sisk, C.L. and Zehr, J.L. (2005) Pubertal hormones organize the adolescent brain and behavior. Front. Neuroendocrinol. 26, 163-174.

85.

1 Pfeifer, J.H. and Allen, N.B. (2012) Arrested development? Reconsidering dualsystems models of brain function in adolescence and disorders. Trends in cognitive sciences $16,322-329$

2 Pfeifer, J.H. and Allen, N.B. (2016) The audacity of specificity: Moving adolescent developmental neuroscience towards more powerful scientific paradigms and translatable models. Developmental cognitive neuroscience 17 , 131-137

3 Badcock, P., et al. (submitted) The Hierarchically Mechanistic Mind: An evolutionary systems theory of the brain and behaviour. .

4 Badcock, P.B. (2012) Evolutionary systems theory: A unifying meta-theory of psychological science. Review of General Psychology 16, 10

5 Friston, K. (2010) The free-energy principle: a unified brain theory? Nature Reviews Neuroscience 11, 127-138

6 Anderson, M.L. (2014) After phrenology. MIT Press

7 Clark, A. (2015) Surfing uncertainty: Prediction, action, and the embodied mind. Oxford University Press

8 Lickliter, R. and Honeycutt, H. (2003) Developmental dynamics: toward a biologically plausible evolutionary psychology. Psychological bulletin 129, 819 
741

742

743

744

745

746

747

748

749

750

751

752

753

754

755

756

757

758

759

760

761

762

763

764

765

766

767

768

769

770

771

772

773

774

775

776

777

778

779

780

781

782

783

784

785

786

787

788

9 Clark, A. (2013) Whatever next? Predictive brains, situated agents, and the future of cognitive science. Behavioral and Brain Sciences 36, 181-204 10 Bastos, A.M., et al. (2012) Canonical microcircuits for predictive coding. Neuron 76, 695-711

11 Friston, K.J., et al. (2010) Action and behavior: a free-energy formulation. Biological cybernetics 102, 227-260

12 Friston, K. (2013) Life as we know it. Journal of the Royal Society Interface 10, 20130475

13 Barrett, L.F. and Simmons, W.K. (2015) Interoceptive predictions in the brain. Nature Reviews Neuroscience 16, 419-429

14 Barrett, L.F., et al. (2016) An active inference theory of allostasis and interoception in depression. Phil. Trans. R. Soc. B 371, 20160011

15 Seth, A.K. and Friston, K.J. (2016) Active interoceptive inference and the emotional brain. Phil. Trans. R. Soc. B 371, 20160007

16 Chekroud, A.M. (2015) Unifying treatments for depression: an application of the Free Energy Principle. Frontiers in psychology 6

17 Joffily, M. and Coricelli, G. (2013) Emotional valence and the free-energy principle. PLoS Comput Biol 9, e1003094

18 Moutoussis, M., et al. (2014) Bayesian inferences about the self (and others):

A review. Consciousness and cognition 25, 67-76

19 Adams, R.A., et al. (2016) Computational Psychiatry: towards a

mathematically informed understanding of mental illness. Journal of Neurology, Neurosurgery \& Psychiatry 87, 53-63

20 Nettle, D. and Bateson, M. (2012) The evolutionary origins of mood and its disorders. Current Biology 22, R712-R721

21 Caporael, L.R. (2001) Evolutionary psychology: Toward a unifying theory and a hybrid science. Annual review of psychology 52, 607-628

22 Geary, D.C. and Bjorklund, D.F. (2000) Evolutionary developmental psychology. Child development 71, 57-65

23 Kenrick, D.T., et al. (2002) Dynamical evolutionary psychology: Mapping the domains of the new interactionist paradigm. Personality and Social Psychology Review 6, 347-356

24 Ploeger, A., et al. (2008) Is evolutionary psychology a metatheory for psychology? A discussion of four major issues in psychology from an evolutionary developmental perspective. Psychological Inquiry 19, 1-18 25 Frankenhuis, W.E., et al. (2013) Bridging developmental systems theory and evolutionary psychology using dynamic optimization. Developmental Science 16, 584-598

26 Tinbergen, N. (1963) On aims and methods of ethology. Zeitschrift für Tierpsychologie 20, 410-433

27 Marshall, P.J. (2013) Coping with complexity: Developmental systems and multilevel analyses in developmental psychopathology. Development and psychopathology 25, 1311-1324

28 Durisko, Z., et al. (2015) An adaptationist perspective on the etiology of depression. Journal of affective disorders 172, 315-323

29 Beck, A.T. and Bredemeier, K. (2016) A unified model of depression integrating clinical, cognitive, biological, and evolutionary perspectives. Clinical Psychological Science, 2167702616628523 
30 Trimmer, P.C., et al. (2015) Adaptive learning can result in a failure to profit from good conditions: implications for understanding depression. Evolution, medicine, and public health 2015, 123-135

31 Gilbert, P. (2006) Evolution and depression: issues and implications. Psychological medicine 36, 287-297

32 Allen, N.B. and Badcock, P.B. (2003) The social risk hypothesis of depressed mood: evolutionary, psychosocial, and neurobiological perspectives. Psychological bulletin 129, 887

33 Vialou, V., et al. (2013) Epigenetic mechanisms of depression and antidepressants action. Annual review of pharmacology and toxicology 53, 59 34 Weissman, M.M., et al. (2005) Families at high and low risk for depression: a 3-generation study. Archives of general psychiatry 62, 29-36

35 Sun, H., et al. (2013) Epigenetics of the depressed brain: role of histone acetylation and methylation. Neuropsychopharmacology 38, 124-137 36 Meaney, M.J. (2001) Maternal care, gene expression, and the transmission of individual differences in stress reactivity across generations. Annual review of neuroscience 24, 1161-1192

37 Heim, C. and Binder, E.B. (2012) Current research trends in early life stress and depression: Review of human studies on sensitive periods, geneenvironment interactions, and epigenetics. Experimental neurology 233, 102-111 38 Gold, P. (2015) The organization of the stress system and its dysregulation in depressive illness. Molecular psychiatry 20, 32-47

39 De Raedt, R. and Koster, E.H. (2010) Understanding vulnerability for depression from a cognitive neuroscience perspective: A reappraisal of attentional factors and a new conceptual framework. Cognitive, Affective, \& Behavioral Neuroscience 10, 50-70

40 Slavich, G.M. and Irwin, M.R. (2014) From stress to inflammation and major depressive disorder: A social signal transduction theory of depression.

Psychological bulletin 140, 774

41 Silk, J.S., et al. (2012) Why do anxious children become depressed teenagers? The role of social evaluative threat and reward processing. Psychological medicine 42, 2095-2107

42 Andersen, S.L. and Teicher, M.H. (2008) Stress, sensitive periods and maturational events in adolescent depression. Trends in neurosciences $31,183-$ 191 43 Gotlib, I.H. and Hammen, C. (2014) Handbook of depression (3rd edn.). Guilford Press 44 Vrshek-Schallhorn, S., et al. (2014) Refining the candidate environment interpersonal stress, the serotonin transporter polymorphism, and geneenvironment interactions in major depression. Clinical Psychological Science 2, 235-248

45 Cohen, S. and Wills, T.A. (1985) Stress, social support, and the buffering hypothesis. Psychological bulletin 98, 310

46 Hawkley, L.C. and Capitanio, J.P. (2015) Perceived social isolation, evolutionary fitness and health outcomes: a lifespan approach. Phil. Trans. R. Soc. $B$ 370, 20140114

47 Leppänen, J.M. (2006) Emotional information processing in mood disorders: a review of behavioral and neuroimaging findings. Current opinion in psychiatry 19, 34-39 
48 Hankin, B.L., et al. (2010) Corumination, interpersonal stress generation, and internalizing symptoms: Accumulating effects and transactional influences in a multiwave study of adolescents. Development and psychopathology 22, 217-235 49 Moore, M.T. and Fresco, D.M. (2012) Depressive realism: a meta-analytic review. Clinical psychology review 32, 496-509

50 Forgas, J.P. (2016) Can Sadness Be Good for You? Australian Psychologist 51 Pizzagalli, D.A. (2014) Depression, stress, and anhedonia: toward a synthesis and integrated model. Annual review of clinical psychology 10, 393

52 Hagen, E.H. (2011) Evolutionary theories of depression: a critical review. The Canadian Journal of Psychiatry 56, 716-726

53 Hames, J.L., et al. (2013) Interpersonal processes in depression. Annual review of clinical psychology $9,355-377$

54 Sloman, L. and Gilbert, P. (2000) Subordination and defeat: An evolutionary approach to mood disorders and their therapy. Routledge 55 Price, J.L. and Drevets, W.C. (2012) Neural circuits underlying the pathophysiology of mood disorders. Trends in cognitive sciences 16, 61-71 56 Kupferberg, A., et al. (2016) Social functioning in major depressive disorder. Neuroscience \& Biobehavioral Reviews 69, 313-332

57 Rushworth, M.F., et al. (2013) Are there specialized circuits for social cognition and are they unique to humans? Current opinion in neurobiology 23, 436-442

58 Davey, C.G., et al. (2008) The emergence of depression in adolescence: Development of the prefrontal cortex and the representation of reward. Neuroscience \& Biobehavioral Reviews 32, 1-19 59 Friston, K., et al. (2015) Active inference and epistemic value. Cogn Neurosci, $1-28$

60 Friston, K., et al. (2014) The anatomy of choice: dopamine and decisionmaking. Phil. Trans. R. Soc. B 369, 20130481

61 Paulus, M.P. and Stein, M.B. (2006) An insular view of anxiety. Biological psychiatry 60, 383-387

62 Pezzulo, G., et al. (2015) Active Inference, homeostatic regulation and adaptive behavioural control. Progress in neurobiology 134, 17-35 63 Waselus, M., et al. (2011) Collateralized dorsal raphe nucleus projections: a mechanism for the integration of diverse functions during stress. Journal of chemical neuroanatomy 41, 266-280

64 Pellicano, E. and Burr, D. (2012) When the world becomes 'too real': a Bayesian explanation of autistic perception. Trends in cognitive sciences 16, 504510 65 Fletcher, P.C. and Frith, C.D. (2009) Perceiving is believing: a Bayesian approach to explaining the positive symptoms of schizophrenia. Nat Rev Neurosci. $10,48-58$ 66 Friston, K.J., et al. (2014) Computational psychiatry: the brain as a phantastic organ. The Lancet Psychiatry 1, 148-158

67 Cuijpers, P., et al. (2011) Interpersonal psychotherapy for depression: a metaanalysis. American Journal of Psychiatry 168, 581-592 68 Park, H.-J. and Friston, K. (2013) Structural and functional brain networks: from connections to cognition. Science 342, 1238411 69 Hohwy, J. (2013) The predictive mind. Oxford University Press 
886

887

888

889

890

891

892

893

894

895

896

897

898

899

900

901

902

903

904

905

906

907

908

909

910

911

912

913

914

915

916

917

918

919

920

70 Sporns, O. and Betzel, R.F. (2016) Modular brain networks. Annual review of psychology 67, 613-640

71 Buckner, R.L. and Krienen, F.M. (2013) The evolution of distributed

association networks in the human brain. Trends in Cognitive Sciences 17, 648-

665

72 Finlay, B.L. and Uchiyama, R. (2015) Developmental mechanisms channeling cortical evolution. Trends in neurosciences 38, 69-76

73 Anderson, M.L. and Finlay, B.L. (2014) Allocating structure to function: the strong links between neuroplasticity and natural selection. Frontiers in human neuroscience 7,918

74 Mengistu, H., et al. (2016) The evolutionary origins of hierarchy. PLOS Comput Biol 12, e1004829

75 Hilgetag, C.C. and Hütt, M.-T. (2014) Hierarchical modular brain connectivity is a stretch for criticality. Trends in cognitive sciences 18, 114-115

76 Hesse, J. and Gross, T. (2014) Self-organized criticality as a fundamental property of neural systems. Frontiers in Systems Neuroscience 8, 46-59

77 Sowell, E.R., et al. (2003) Mapping cortical change across the human life span. Nature neuroscience 6, 309-315

78 Blakemore, S.-J. (2012) Imaging brain development: the adolescent brain.

Neuroimage 61, 397-406

79 Spear, L.P. (2000) The adolescent brain and age-related behavioral manifestations. Neuroscience \& Biobehavioral Reviews 24, 417-463

80 Fuhrmann, D., et al. (2015) Adolescence as a sensitive period of brain development. Trends in cognitive sciences 19, 558-566

81 Blakemore, S.-J. and Mills, K.L. (2014) Is adolescence a sensitive period for sociocultural processing? Annual review of psychology 65, 187-207

82 Crone, E.A. and Dahl, R.E. (2012) Understanding adolescence as a period of social-affective engagement and goal flexibility. Nature Reviews Neuroscience 13, 636-650

83 Brown, B.B. and Larson, J. (2009) Peer relationships in adolescence. In Handbook of Adolescent Psychology. (Lerner, R.M. and Steinberg, L., eds), pp. 74103, John Wiley \& Sons

84 Sisk, C.L. and Zehr, J.L. (2005) Pubertal hormones organize the adolescent brain and behavior. Frontiers in neuroendocrinology 26, 163-174 86. 